\title{
A Model for Visual Perception and Recognition of Outlines
}

\section{David Firth}

20 September 2017

\section{https://psyarxiv.com/fxpq5}

\section{Affiliation: none e-mail: dfirth001@pobroadband.co.uk}

\begin{abstract}
Many topics in vision are still not well understood. A model for visual perception and recognition of shapes is described, by combining ideas relating to receptive fields of ganglion cells in the retina, eye movements during fixation, multiple spatial scales of precision, attention, axes and coordinates. The main objective is to provide a realistic scheme for perception of outlines, with recognition that is not prevented by changes of size and viewpoint. Outlines are detected by changes with time arising from eye movements, followed by identification of vertical and horizontal axes, and use of levels of precision that depend on the size of the area of attention. The increments of position in these levels increase in size as the size of area increases, so in an index of positions the increment numbers remain constant for all sizes. After changes of viewpoint, "attentional planes" connect changed positions on outlines to positions with a standard orientation of axes, so that shapes may be matched with stored representations. Spatial coordinates are related to positions at the retina by representations based on eye movements.
\end{abstract}

Key words: vision, perception, recognition, attention, axes, viewpoint 


\section{Introduction}

Visual recognition of shapes occurs frequently and usually with such ease that we might suppose the procedure producing recall to be reasonably straightforward. However, investigations to date have shown that there are many problems, and there is still only a rather limited understanding of them from theories and experiments. Limiting the scope of an investigation simplifies matters considerably, but the result obtained may turn out to be incompatible with the wider requirements. The aim here, and the justification, is to put together some of the ideas that have not often been combined in the past, in a model for recognition of three dimensional objects from various viewpoints, but excluding binocular effects, shading and texture. Even with these restrictions, the problems addressed include perception and early vision, attention, invariance of recall with changes of size and viewpoint, and scenes containing several objects. It is a model, not science, but is intended to be realistic, so that everything contained in it could reasonably be expected to be present in our own processing of visual information. Consequently, it comes into the "connectionist" category, in which representations are formed in a network containing a large number of cells with connections between them. There is no external agency to control the flow of activity, so the procedure is the same for storage and recall, the only difference being whether the representations are new or already stored.

Marr (1982) produced a computational model addressing what was represented in human vision, and why it was required. The model described here is intended to show how representations can be organized, in a way that is more natural than computation. For edge detection and representations Marr started by smoothing intensity over spatial fields of a number of sizes, and that approach is used here, in groups and levels. In the description which follows the term intensity will be used for magnitude of stimulus.

The model starts with outline detection using change in intensity as a result of movements, then vertical and horizontal axes in levels of precision that depend on the size of the area of attention, and viewpoint invariance from attentional planes that connect positions in rotated axes with positions before rotation, to allow shapes to be matched with stored representations. Mental rotation was investigated experimentally by Tarr \& Pinker (1989); here it is achieved by selecting an attentional plane that was learned previously. In each level, absolute positions of outlines in viewer centred coordinates are in low precision - primary $\boldsymbol{V H}$ positions - with higher precision used to obtain angles of small pieces of outline relative to the orientation of an axis of the object. Levels of precision, coordinate systems and oriented edge segments were part of Marr's theory, which also contained a number of topics included here only briefly or not at all, such as surfaces and depth, representations in three dimensions and stereopsis.

In the theory of Biederman (1987) the main emphasis is on a later stage of vision than is being modelled here - the procedures precede Biederman's volumetric primitives, called "geons". An object is a simple shape or piece of outline, which is at the same stage as the "nonaccidental properties", such as "curved" or "straight" that are attributes of geons. A major difference is that geons are invariant with viewpoint, whereas here viewpoint invariance is obtained using attentional planes; a feature of Biederman's theory that is used here is that stored representations are in two dimensions.

Ahissar \& Arieli (2001, 2012) used spatial coding for coarse, absolute positions, and temporal coding for fine detail using relative positions. That approach for positions is used here, except that relative positions, in the form of traces, are from spatial distributions; traces are used down to a size of area of attention of 20 ' radius, approximately - they are not suitable for "vernier acuity", i.e. resolution of detail smaller than the separation of receptors, so "hyperacuity" is excluded. 
An introduction to the problems in recognition can be found in Pinker (1984), and books that were used are those of Bruce, Green \& Georgeson (2003), Howard (1982), Schiffman (2001) and Gross (2001).

\section{Groups}

The model of perception is based on points in the retinal image, and a visual plane containing the vertical and horizontal components of motor activity that produces eye movements to equivalent points in the visual field.

Experiments suggest that ganglion cells in the retina have approximately circular receptive fields of receptors, and that their responses depend on how an outline is situated with respect to two areas within them, circular centres and annular surrounds; reviews can be found in Bruce, Green \& Georgeson (2003) and Howard (1982). In the present model detection of outline occurs within these receptive fields, i.e. within groups of receptors. The centres are used for positions in the perception, the centres and surrounds together for storage and recall of shapes, and for representations of intensity. Centres also provide a limited quantity of precise storage and recall of shapes.

\section{$3 \mathrm{VH}$ positions and traces}

Perception and recognition of shapes requires some form of representation of positions on outlines, one that is compatible with eye movements, curvature of the retina and invariance of recall with size and viewpoint. A small quantity of connected outline, for example a single letter of text or handwriting, is called an object. A scene consists of more than one object, connected or unconnected.

Groups of receptors are linked to representations of objects that contribute to the perception that is seen. An associated set of spatial coordinates is based on motor activity that produces eye movements to positions on outlines. When this activity is stored and recalled it represents knowledge of the perception seen.

It is necessary to suppose that motor activity has two components, one giving the distance, angle and increment for the movement, the other causing the actual activation of muscle fibres prescribed by the first component. The representations of position that are stored and recalled are in the same form as the first component - motor activity without the "do it" element, rather like an imaginary trial of an action, or movement of the position of attention. The degree to which these are visual or motor activity representations is discussed further in Section 18. This is essentially the approach expressed by Helmholtz (1867), which included the descriptions "degree of innervation which we cause to be communicated to the nerves of the ocular muscles", and "intensity of the effort of will".

Representations of position in the perception are obtained from angles of rotation of the forward axis relative to the observer, in elevation vertically and in azimuth horizontally, as shown in Figure 1 . The representations are $\tan (\psi), \tan (\varphi)$, where $\psi$ (psi) and $\varphi$ (phi) are angles of elevation and azimuth respectively. In order that they can be described in a way that is more consistent with experience than rotations, positions are considered to be in the visual plane, illustrated in Figure 2, which is perpendicular to the forward axis, and the visual centre is the origin of axes; also shown are polar coordinates $\mathrm{r}, \theta$, which are used in Section 10. Positions in the visual plane are used for stored representations of shape. Regions of the visual plane that are not close to the visual centre collectively form the periphery. Positions have rectangular Cartesian coordinates $\boldsymbol{V}, \boldsymbol{H}$, and they are referred to henceforth as $\boldsymbol{V H}$ positions in normal axes $\boldsymbol{V}, \boldsymbol{H}$, which are discussed further in Section 13. Coordinates in the visual plane are shown using italic notation, to indicate the practice of representing them by tangents of their angles of elevation and azimuth. Representations need to be in angles to be realistic, particularly for three dimensional scenes and 
perspective, but in many respects modelling and discussion are simplified by use of intersections of "viewing directions" with a visual plane. Use of $\tan (\psi), \tan (\varphi)$ provides values that are proportional to position in the visual plane, and also includes effects of perspective. So, eye movements cause objects to move relative to the visual centre, with stored representations that are essentially the same as those of $\boldsymbol{V H}$ positions. In terms of the coordinates of the perpendicular projection, $\mathbf{v}, \mathbf{h}$, onto the visual plane, $\mathbf{v} / \mathbf{D}=\tan (\psi)=\boldsymbol{V}, \mathbf{h} / \mathbf{D}=\tan (\varphi)=\boldsymbol{H}$, where $\mathbf{D}$ is the distance coordinate along the forward axis. Foley, Ribeiro-Filho, \& Da Silva (2004) carried out experiments to determine how well reported perceived size and distance matched values obtained using $\mathbf{v} / \mathbf{D}, \mathbf{h} / \mathbf{D}$; they identified discrepancies and suggested a modification that would improve the agreement - see Section 16 for further comments on size.

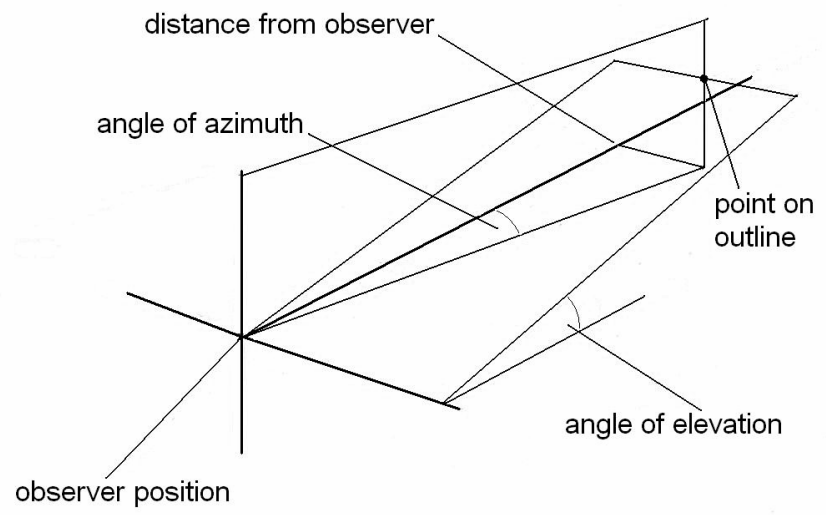

Figure 1 Angles of elevation and azimuth of a point on an outline.

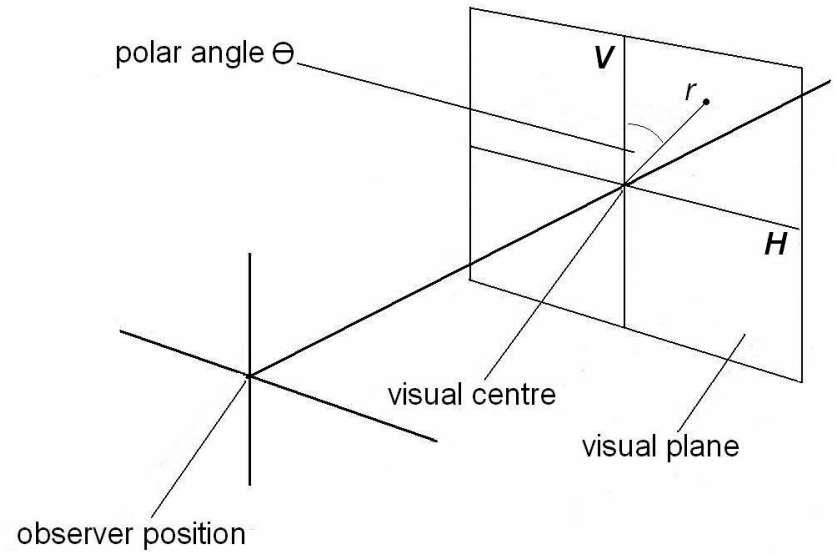

Figure 2 Visual plane with rectangular coordinates $V, H$, and polar coordinates $r, \theta$ of a point.

A piece of continuous, smooth outline, along which there are no sudden changes of direction, is called a length. The magnitudes of lengths in shapes that have not been recalled determine interest, and the next area of attention is the region with highest interest - that would usually contain the largest unknown outline.

Lengths are terminated by ends of outline, or by junctions with other lengths, or by points at which there are changes of direction of $\boldsymbol{V}$ or $\boldsymbol{H}$; all of these are ends of lengths. For changes of direction of $\boldsymbol{V}$ or $\boldsymbol{H}$ the direction in which the end is facing can also be used. The following description is for stationary objects, so reference to movements of objects means apparent changes of position arising from movements of the eye, i.e. movements of the visual centre.

$\boldsymbol{V H}$ positions become linked to positions of groups, by movements of localized features, such as ends of lengths of outline. The movements would be along approximately straight lines, passing between the position of a group and the visual centre, as the centre moves to or from the localized features; this is shown in Figures 3 and 4. 


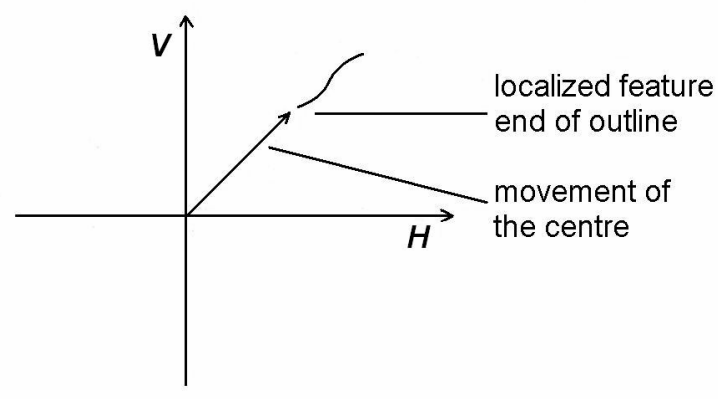

Figure 3 Movement of the visual centre to an end forms a link between $V H$ positions in the visual plane and positions at the retina.

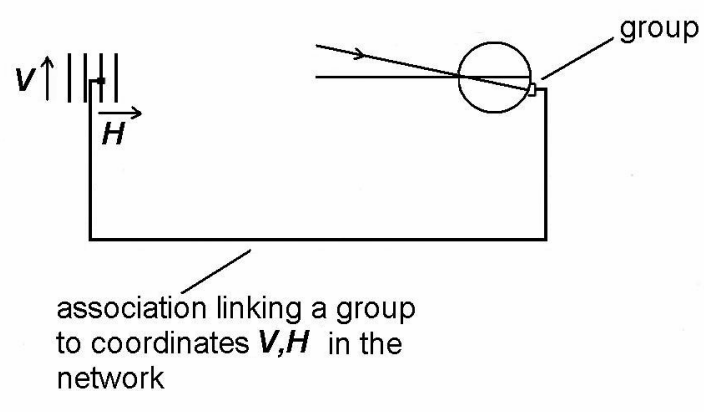

Figure 4 Connections are formed between positions of groups and representations of coordinates $V, H$ in the network of cells.

Objects vary considerably in size - consequently, both the ends of lengths of outline and the movements occur at a number of levels of precision in the visual plane, as illustrated in Figure 5. $\boldsymbol{V H}$ positions are used only within $\boldsymbol{V H}$ regions for which movements are repeatable with a certain precision, and which have sizes that increase with size of increment. A level consists of a primary $\mathbf{V H}$ area with approximately 40 primary increments radially, in which each of the $\boldsymbol{V H}$ positions has a primary increment consisting of approximately 7 smaller, secondary increments, shown in Figure 6. The term "radially" will be used to indicate directions in which increments change along $\boldsymbol{V}$ or $\boldsymbol{H}$ or along both $\boldsymbol{V}$ and $\boldsymbol{H}$. Further description of groups is given in Section 8.

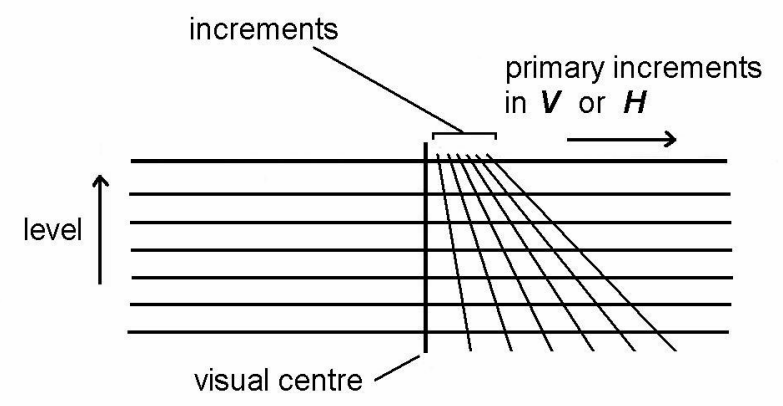

Figure $5 \mathrm{VH}$ positions in the visual plane have many levels of precision, with size of increment decreasing as level increases.

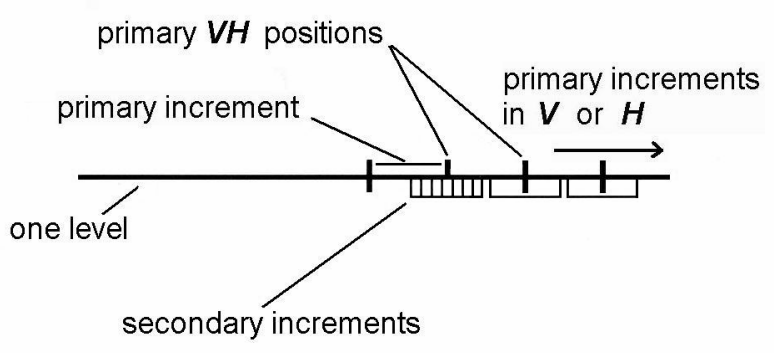

Figure 6 Each primary increment of $\mathrm{VH}$ positions contains a number of smaller secondary increments, shown here for a single level.

Each primary increment contains a set of traces, which are representations of angles of outline in the visual plane, with the precision of secondary $\boldsymbol{V H}$ positions. They are paths traced out locally on the retina by features, such as "spots" or ends of outline, during small movements. For most positions, traces can be obtained with greater precision than is possible for movements to them from the centre. A single trace value represents an angle of the same value occurring anywhere within the primary increment; there is uncertainty in the position of the element of outline. So, outlines are represented by primary $\boldsymbol{V H}$ positions, each of which has a set of traces that represent possible angles of outline somewhere within the primary increment; this is illustrated in Figure 7 - primary groups are defined in Section 8. Resolution of traces is approximately $8^{\circ}$, but is improved by use of differences of responses, to give angles of outline. A scheme 
of this kind is given by Howard (1982) pp.111-113, using a principle similar to that of the opponent process theory for colour.

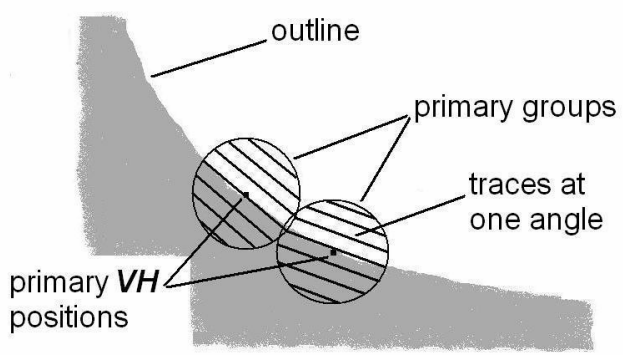

Figure 7 Two primary VH positions and trace values on an outline.

When the perception is set at a particular level, i.e. for a particular size of object, the primary $\boldsymbol{V H}$ positions and traces at that level are used. The level at which the perception is set changes continually. Most storage and recall occurs at the precision of primary $\boldsymbol{V H}$ areas - see also Sections 6 and 13. When attention moves to the periphery recall can occur there.

It is likely that angle of outline is an important property in visual representations - a description of the evidence from experiments has been given by e.g. Bruce, Green \& Georgeson (2003). Traces, junctions and ends of outline are similar to properties in the approach of Marr (1982), where "place tokens" included oriented edges, points of discontinuity in orientation and terminations. Coarse and fine orientations for geons were used by Hummel \& Biederman (1992).

\section{Levels}

A feature of the approach of Marr (1982) was the combining of small and large spatial scales, and that principle is used in the model being described here. The spacing of $\boldsymbol{V H}$ positions varies, depending on the size of object for which the perception is set. These increments in $\boldsymbol{V H}$ position are used in, and define, levels of the visual plane, stored representations and the perception. High levels contain small increments, and lower levels contain progressively larger increments. The increment is constant within a level. Each level applies only within a fixed radial distance from the centre of the visual plane.

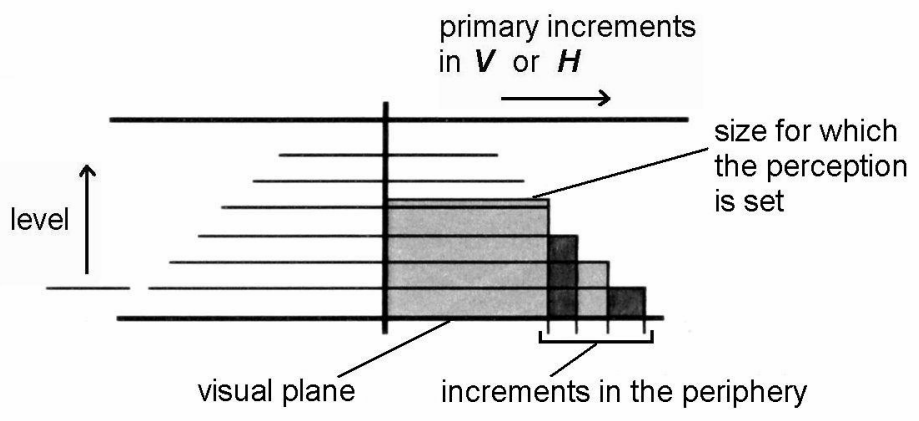

Figure 8 The size of area for which the perception is set determines the level. 
Across levels, the increments furthest from the centre form a continuous sequence, in which their sizes increase as radial position increases - see Figure 8 . The radial size of levels increases progressively from high level to low. Stored representations of objects are also in levels, usually in several levels.

\section{Attention}

The level at which the perception is set and the position, size and rotation of the region for which storage or recall of shape occurs define the term attention. A duplicate of the visual plane, sometimes with a translational shift applied, is called an attentional plane, shown in Figure 9. The size of the area of attention determines the level, for both the perception and stored or recalled representations. Attention allows storage of outlines in selected regions. Each time the perception is set, store or recall follows something is known about the shapes that have been observed, either new stored knowledge or recall of existing knowledge. This representation is of the same type as an "idea" or "memory-image", described by Helmholtz (1867) as "the image of visual objects as retained in the memory without being accompanied by any present sense-impressions".

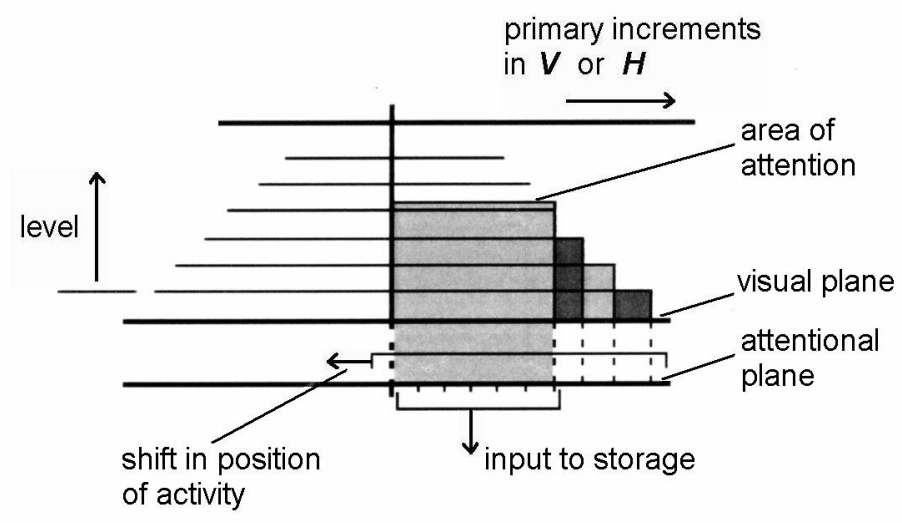

Figure 9 An attentional plane is connected to increments in levels of the visual plane. Increments in the attentional plane within the area that is set are connected to storage. A shift in position of attention may be applied to allow recall from activity in the periphery.

So, 'known' is defined as a reference to the representations that have been stored. 'Seen' is more complex it is defined here as the perception before storage or recall. That could occur, for example, in the periphery of a new scene with attention at the centre, or, while observing a page of text without recall of every word. However, it is possible that there would be some knowledge, but not enough for recall of shapes, perhaps whether light or dark or having outlines present. In the model being described recall is mainly within the area of attention, and the terms are used despite their limitations.

Increments in the attentional plane are connected to storage. Stored representations are activated from whatever area of attention is currently set, but, as will be shown later, they are invariant with level. Objects in the periphery may be recalled by shifting attention; in the model this is shown as a shift in the position of activity in the attentional plane, so that the input to storage can remain in a fixed position, which seems to be a simpler concept than having a moving input. It should be noted that eye movements cause the activity in the visual plane to move relative to the centre, so two types of shift are possible. While movement of an object into the periphery is a frequent occurrence, it is likely that shifts of attention are unusual - most storage and recall would occur with attention at the visual centre. 


\section{Storage and recall of shapes - preliminary description}

Storage and recall of shapes occurs at the current attentional centre. Stored representations of outlines contain their primary $\boldsymbol{V} \boldsymbol{H}$ positions in $\boldsymbol{V}, \boldsymbol{H}$, but not at the full resolution. The maximum number of $\boldsymbol{V} \boldsymbol{H}$ positions stored radially is usually $7-10$, perhaps up to 20 , approximately, of the 40 in the perception seen. When the perception is set at the highest level for storage of an object of a particular size, the maximum number of positions is stored. That is achieved with an area of attention of the same size as the object. But the level for storage is well below the level of the perception seen, as shown in Figure 10. Before reduction to the smallest size, representations are stored using larger areas of attention with larger increments, with consequent reductions in the numbers of $\boldsymbol{V H}$ positions, perhaps only two or three. In this way, shapes of objects are stored using multiple levels of representation. The highest level reached depends on the time for which the observation is made - higher levels with more stored $\boldsymbol{V} \boldsymbol{H}$ positions require longer periods of time. When the object is smaller than the area of attention for which the perception is set fewer positions along its outline would be stored.

Storage of representations with precision greater than that of primary $\mathbf{V H}$ positions is described in Section 13.

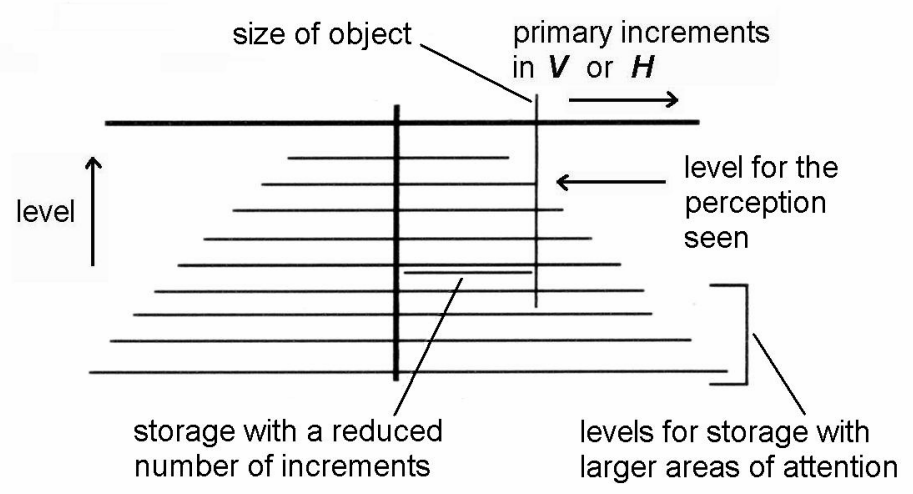

Figure 10 The level for the perception seen is higher than the highest level stored. Representations are stored in a number of levels as the size of area decreases.

When the attentional centre is in the periphery, the perception of an object at that position may contain fewer increments and $\boldsymbol{V H}$ positions than would be used for storage at the visual centre. In that case recall may be achieved by use of lower levels, so that fewer increments and $\boldsymbol{V H}$ positions are used.

\section{Perception}

Perception of outlines occurs in two stages. The first, or early, stage is perception within an overall area of attention, before storage or recall of objects has occurred, i.e. before an area of attention has been set. When the size of the area has been less than the whole of the visual plane, some regions may contain stored or recalled shapes and others may contain an early perception. Objects in the second stage of perception are known, because there is an awareness of objects not present in the first stage. 


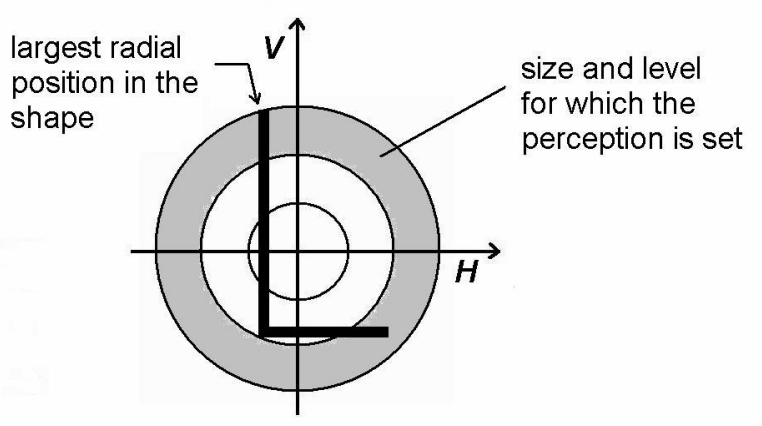

Figure 11 Illustration of three levels when the perception is set for the object ' $L$ '.

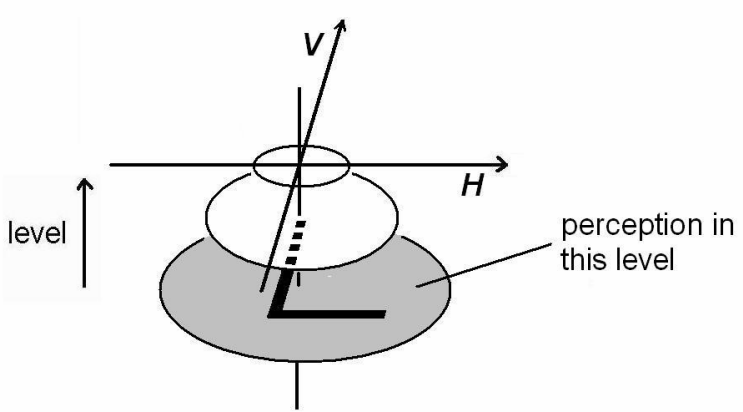

Figure 12 The same case as in Figure 11, showing the visual plane in two dimensions.

Following the early stage, there is an awareness of the scene that is usually less precise than shapes of objects, and a representation of it would be stored - it would be storage with an area too large for recall of objects Usually, but not necessarily, the area of attention is at the centre of the visual plane. After storage or recall an object usually moves to the periphery, into lower levels, outside the area of attention. Any change in an object's axes or shape after movement to the periphery would not be known, unless attention moved there. Figures 11 and 12 show a number of levels, including the level for which the perception is set when observing an object, and Figure 13 illustrates movement of attention into the periphery.

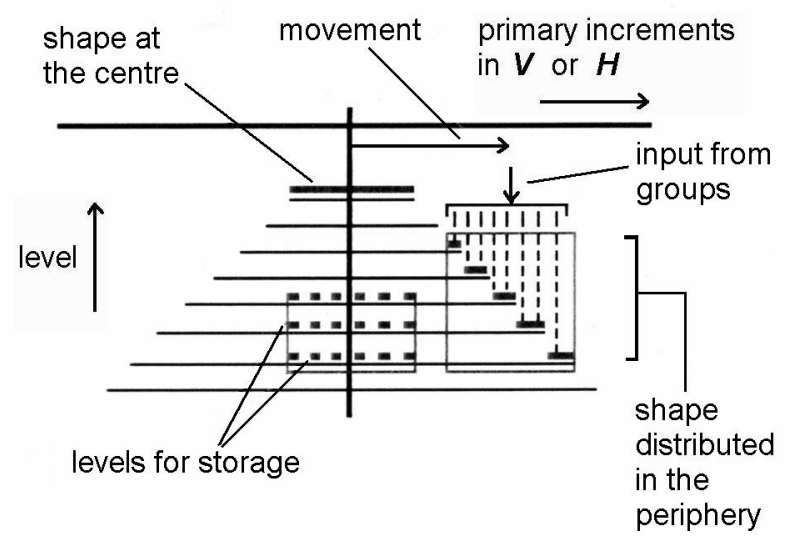

Figure 13 Storage or recall with an object at the centre, with the possibility of movement of attention to the periphery for recall of an object there, at a lower level if necessary.

Small objects are usually stored or recalled using a small area of attention, in which increments are small, at the centre of the visual plane. Outside the small area objects are in lower levels; they are also in lower levels within the area of attention. Storage of shapes in multiple levels allows recall with a match that does not need to be exact, and also permits recall using fewer increments when an object has moved to the periphery. Level depends on the highest radial position in the area of attention. 


\section{Detection of sharp outlines for the perception and stored shapes}

$\boldsymbol{V H}$ positions and secondary increments in the perception are related to positions and sizes of centres of groups of receptors. For storage and recall of shapes, $\boldsymbol{V H}$ positions and primary increments used are related to positions and sizes of groups. Groups of different sizes overlap, in a way that is consistent with levels of $\boldsymbol{V H}$ positions.

The response of a group is a positive value. When reduction or subtraction is required it is obtained by use of cells with two types of input, one that causes activation, and the other inhibition of its response. A group has a large positive response when the whole of an outline falls within its centre region, or when a continuous outline crosses both the centre and surround.

Smaller positive responses occur when no outline exists within a group, or when an outline falls within or crosses a surround - they represent blurred outlines or intensity. These cases are shown diagrammatically in Figure 14.

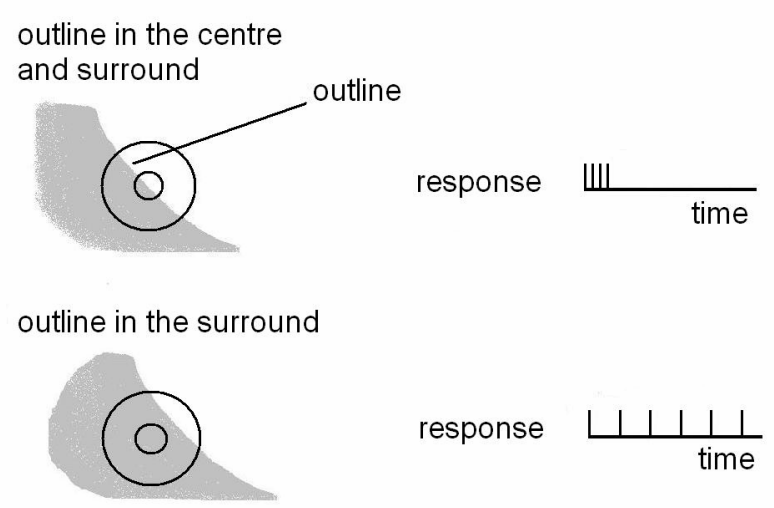

Figure 14 Representations for outline and intensity. Top: outline in a centre causes a transient large response, here shown as a rate of firing. Bottom: no outline in the centre gives a smaller response, representing a blurred outline, described in Section 14, or intensity.

Groups containing all the centres reached by a primary $\boldsymbol{V H}$ position representation are called primary groups. So a primary group contains many centres in addition to its own, positions that could be reached by the movement corresponding to the primary increment associated with that group, because of the limitation on precision. In effect there is a grid of primary $\boldsymbol{V H}$ positions.

A secondary $\boldsymbol{V H}$ position on an outline corresponds to the centre of a group that has a large response; an outline crossing the centre of one group would also be in the surrounds of neighbouring groups. Centres and secondary $\boldsymbol{V H}$ positions are used for traces, primary groups and primary $\boldsymbol{V} \boldsymbol{H}$ positions are used for storage and recall. Primary $\boldsymbol{V H}$ positions and traces, with some further developments described in Section 13, form the outline representation in the perception seen.

Detection of an outline in the centre of a group occurs as a result of movements, which produce changes of intensity with respect to time at the positions of receptors. During fixation, slow drifts of the eye, and any other slow movements, would be suitable. Saccades and microsaccades, as described by Howard (1982), are not used because of uncertainty in position, and the possibility that they do not 
contribute to visual perception, except for movement of the visual centre to new points of fixation saccades would be for new, well-separated objects and microsaccades for smaller shifts in position when scanning within an object, as a connected scene of smaller "parts". Further details of outline detection are given in Section 11, and recall of blurred outlines is described in Section 14.

Groups within a single level increase in size with increasing radial position, but they decrease in size as level increases. It is possible for there to be a degree of overlap between groups within a single level, for example by half a group radius. The overlap would be in the groups of receptors, not in the primary increments. In order to avoid problems at the edges of increments, traces can be considered to be defined over the visual plane, in parallel with the set of primary $\boldsymbol{V H}$ positions; then, with overlapping groups, the primary $\boldsymbol{V H}$ position used would be the one closest to the trace most strongly activated.

\section{Storage of shape representations of objects}

Shape representations are stored after movement of attention to the object, usually when the object is at the visual centre. That occurs when the distribution of $\boldsymbol{V H}$ positions of ends of lengths is balanced with respect to the visual centre, which is an approach suggested by Pinker (1984), using "visual center of mass" as an origin for axes. Hummel \& Biederman (1992) used the centroid of a geon to define its location. This defines the "centre" of an object. Increment numbers from the primary $\boldsymbol{V H}$ areas in levels are connected to storage. Corresponding increment numbers in adjacent levels are linked, by a procedure that permits activity to transfer from one level to the next with a change of position, equivalent to a magnification or reduction in size, while maintaining constant increment numbers. That provides recall that is invariant with size. The increment numbers, which apply for all levels, can be viewed as forming a single plane of increment numbers. Stored representations are the "positions" in that plane, as shown in Figure 15. Storage usually occurs with more precision than recall; that occurs naturally by use of levels, by storing initially with a smaller area of attention than is usually used for recall.

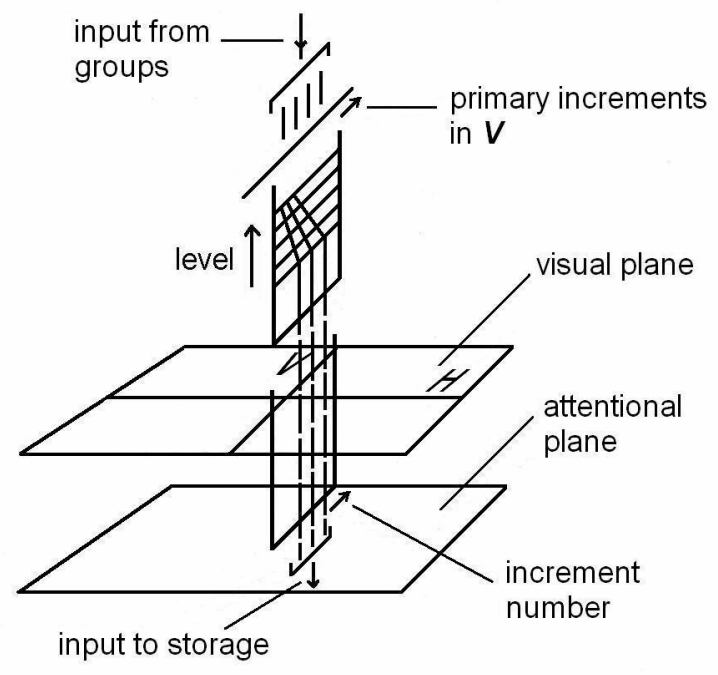

Figure 15 Illustration of links across levels of corresponding increment numbers, and their connection to storage; only increments along the $V$ axis are shown. 


\section{Invariance of recall with change of viewpoint}

The first principle used is that the perception is partly the shape that is seen and partly knowledge of it. When the viewpoint changes the knowledge of it remains unchanged. There are a number of attentional planes, created by making movements to positions using increments applied not along the directions of axes $\boldsymbol{V}, \boldsymbol{H}$, but along rotated $\boldsymbol{V}, \boldsymbol{H}$. The visual planes formed in this way would serve as attentional planes. However, formation of links between increment numbers and primary groups involves a movement to every position in every attentional plane, so an alternative approach is required. When an object rotates, with active increment numbers on its outline held fixed, identifiable positions, such as ends of lengths of outline, could be used to determine the primary groups for primary $\boldsymbol{V H}$ positions in attentional planes for the rotated axes of the object. With this approach there is a difficulty that has to be overcome, that of forming links from increments to the correct groups on the outline when many are active at the same time - no problem arises when only one identifiable position is active, but in that case the number of repetitions of the procedure required may be unrealistic. Despite this reservation, use of multiple positions seems to be the most suitable method of obtaining links from increment numbers to primary groups in the large number of attentional planes required, particularly if links could be formed for positions along lengths as well as at ends.

Although representations are in the form of increments along axes that are vertical and horizontal they would arise from an ability initially to move to a position of interest $r, \theta$ in the visual plane, where $r$ and $\theta$ are radial and circumferential polar coordinates. A set of representations $F_{n}$ would cause movement with a particular size of radial increment to a position of interest $r, \theta_{n}$ in the visual plane, where integer $n$ denotes a number in the set; the radial position reached would depend on the number of increments used. The $F_{n}$ would be motor activity representations, each producing movement to a particular group, using muscle fibres in nominally "vertical" and "horizontal" directions. The two representations from $F_{n}$ in the directions of $\boldsymbol{V}$ and $\boldsymbol{H}$ would produce movements to $\boldsymbol{V H}$ positions along those axes for a specified size of increment and number of increments, defining those $\boldsymbol{V H}$ positions. Superposition of movements along those axes would define all other $\boldsymbol{V H}$ positions in a quadrant of the visual plane, giving the same results as when obtained using $\mathrm{F}_{\mathrm{n}}$ directly.

Consider how attentional planes could arise: first of all, perhaps, several different representations would be stored; eventually the number required would become large, in which case it would be more efficient to create new attentional planes. Increments are linked across, or linked in parallel, to different attentional planes. In an attentional plane with changed positions, the coordinates in the $\boldsymbol{V}, \boldsymbol{H}$ plane are displaced while remaining connected to the same increment numbers. This allows one "storage" plane of increment numbers to correspond with many possible planes of $\boldsymbol{V H}$ positions.

For example, one set of attentional planes would represent rotations in the $\boldsymbol{V}, \boldsymbol{H}$ plane, with a spacing of approximately $3^{\circ}$. When one of those rotations matches the rotation of a shape that has been stored and then rotated, the increment numbers activated in that attentional plane would be the same as in the stored representation. That provides invariance of recall with rotation in the $\boldsymbol{V}, \boldsymbol{H}$ plane.

When connections have been formed from groups in all levels to primary increments in one attentional plane, probably in $\boldsymbol{V}, \boldsymbol{H}$, it is possible to form other attentional planes more efficiently than described so far, by forming links between primary increments in the first attentional plane and primary increments in the new planes. The changed positions would then be in planes of increment numbers instead of groups. The advantage is that one set can be used for all levels - they would be invariant with size. 
Attentional planes need to be selected. When no recall occurs using an attentional plane aligned with $\boldsymbol{V}, \boldsymbol{H}$, the next stage is to identify a new one, by recognition of a suitable cue for that particular viewpoint. When that has been done, the required attentional plane is selected.

Use of attentional planes is a way of achieving mental rotation, a hypothesis investigated experimentally by Tarr \& Pinker (1989), with subjects storing and recalling objects with changed orientations; two possibilities identified there are mental rotation of representations to a standard orientation, and storage and recall of multiple orientations; both of these can be obtained by use of attentional planes. Axes in two dimensions were used in the model of Hummel \& Biederman (1992), represented by "axis cells".

After rotations of an object in three dimensions, the normal axes used for the stored shape, and subjected to the same rotations, may no longer be perpendicular. In that case they are denoted by $\boldsymbol{V}^{\prime}, \boldsymbol{H}^{\prime}$. As well as change in the angle between vertical and horizontal axes, rotation of a shape out of the $\boldsymbol{V}, \boldsymbol{H}$ plane produces a change in the ratio of height to width. The rotation of axes from $\boldsymbol{V}, \boldsymbol{H}$ to $\boldsymbol{V}^{\prime}, \boldsymbol{H}^{\prime}$, and the resulting changed frame of reference, would be angles and axes in projections onto the visual plane. Attentional planes are required for both of these types of change of position. First, however, the directions of the vertical and horizontal axes $\boldsymbol{V}^{\prime}, \boldsymbol{H}^{\prime}$ would be identified. Then, this procedure converts a three dimensional shape into stored representations that are in two dimensions.

The effects of perspective are reasonably small if the size of the object is much less than the distance from the observer, and reduced further when rotations out of the visual plane are also small, so they would usually not be large enough to prevent recall.

All outlines would be in all attentional planes, but only certain outlines would be recalled in each one those for which the changes of position produced a match with a stored representation. To summarize, the outline is the same in all attentional planes; when necessary for storage or recall, use of a different attentional plane allows the spatial grid of increments to be changed. For objects in three dimensions several attentional planes would usually be used; for example, an outline of a cube would have different faces recalled in different attentional planes. This may, perhaps, contribute to an impression of an object having three dimensions.

\section{Representations of small objects at the visual centre}

Clear perception of small objects at the visual centre requires small groups with sizes that are reasonably similar to sizes of the primary increments in their representations - similar because groups may be overlapped, and they increase in size radially. It follows that the centres of groups also need to be small. Near the visual centre it is possible to have 7 centres in a primary increment, or group diameter, only for eccentricities greater than a value slightly less than $2^{\circ}$. Therefore, there are some differences in approach outside and within this radius.

The positions of a moving outline need to be obtained from discrete positions of receptors, to give a perception of the object at any point in time. Ahissar \& Arieli (2001) pointed out that small movements of all outlines together are unlikely to be significant - slow drifts of the eye, and many other movements, are sufficiently slow and small in magnitude that we see them without being aware of them, rather than experiencing a blurred outline. Although certain eye movements are not noticed, it is necessary to see movements of objects clearly. At the retina an outline is the set of contiguous receptors that experience a change of intensity with respect to time, assuming that centres of groups are single receptors. At a particular point in time, a piece of the outline may be at the centre of one receptor and another piece at the edge of another. A small time earlier the outline would have been distributed over a different set of 
neighbouring receptors. In order to prevent a blurred outline it is necessary for receptor responses, or the activity resulting from them, to be "switched off" somehow. The simplest approach, possibly, is to use transient responses - lateral inhibition seems more complex. The method of Ahissar \& Arieli $(2001,2012)$ would be suitable, namely onset times of bursts of "spikes"; here "temporal encoding" is not used, so the outline representation would be the occurrence of the first spike at a particular position.

So, positions of outline are required from transient responses. In order that the perception seen can arise from the same type of representation at the visual centre and in the near periphery it is necessary to use primary $\boldsymbol{V H}$ positions and traces. Traces can consist of two or three centres, of one receptor each, obtained from paths on the retina of "spots" or ends of outline during small movements of the eye. The approach is the same as for positions in the periphery, except that centres have one receptor each and groups contain traces with fewer centres. There is a lower limit for the size of the area of attention, a diameter of approximately 40 minutes of arc.

\section{Representations of small "spots"}

A question that has not so far been addressed is how a small spot stimulus can be represented. Traces are not suitable because no angle of outline is involved. A group has an active outline representation when a spot is in its centre. So, the representation for a spot is outline and intensity directly, rather than trace and intensity. The perception of a spot in the periphery would have the precision of primary $\boldsymbol{V H}$ positions in the appropriate level - it could be improved by movement towards the visual centre, with an increase in level. If a small object in the periphery were too small for traces to be formed it would be seen as a spot.

\section{Stored representations using angles of outline}

Stored representations are required with greater precision than is obtained by use of primary $\boldsymbol{V H}$ positions alone - for example, when we recognize a circle or a straight line on a page. Therefore, for some primary $\boldsymbol{V H}$ positions angles of outline are stored as well. Usually these representations are only stored for a few parts of an object, because of a limit on the quantity of storage possible in a single observation. As the number of observations of an object increases, so the number of stored angles increases. The principle is to store only information that is useful, which prevents storage, for example, of every part of a long, uniform object. Angles of outline are present in all parts of the perception seen, however precision also depends on the size of the area of attention, which determines the level for the representations seen and stored.

It is necessary for recall of angles of outline to be invariant with rotation of a shape in the visual plane, i.e. in the plane of axes $\boldsymbol{V}, \boldsymbol{H}$. For small rotations out of the $\boldsymbol{V}, \boldsymbol{H}$ plane recall of precise shape would be impaired, and for large rotations recall using angles would not be possible, because the angles are in projections onto the $\boldsymbol{V}, \boldsymbol{H}$ plane. There is no precise reference direction for angles of outline from traces, because resolution is only $8^{\circ}$, approximately. The only precise direction is provided by outline, within the object or, perhaps, from an adjacent object. So, angles that are stored and recalled are relative angles, with the direction of $\boldsymbol{V}$ or $\boldsymbol{H}$, or $\boldsymbol{V}^{\prime}$ or $\boldsymbol{H}^{\prime}$, as a reference. Representations of angles of outline occur in levels, and contribute to the perception that is seen; relative angles are obtained in attentional planes, used for storage and recall, and form part of the perception that is known. When no suitable outlines exist, directions of axes would be obtained from another source, such as gravity or self, or perhaps from a slightly earlier observation.

A consequence of the absence of any absolute reference direction for axes is that the directions of vertical 
and horizontal axes in the visual plane, $\boldsymbol{V}, \boldsymbol{H}$, are determined in the same way as just described for axes of an object. The perception seen has an uncertain reference direction for angles of outline, which influences all outlines equally. It follows from this that the $\boldsymbol{V}, \boldsymbol{H}$ plane in the absence of rotation is not necessarily any one particular attentional plane - the absolute "coordinates" are the increment numbers in levels.

\section{Detection and recall of blurred outlines}

Shapes can be recalled even when their outlines appear blurred. A large area of attention would allow recall using large primary increments at a low level, but sometimes that is not realistic. So that recall is possible using a reasonably small area of attention, it is necessary to have detection of outlines in surrounds of groups of receptors. For a particular level, this would only occur for outlines that were not sufficiently sharp to be detected in the centres of groups, and a form of response different from that for uniform intensity would probably be required - see also Section 18.2 . Outlines detected in surrounds in this way have no traces, so recall is achieved using the primary $\boldsymbol{V H}$ positions detected, without any precise representations from angles of outline. A sharp outline in a surround would be in the centre of an overlapping group.

\section{Generalized representations}

One of the most important questions in object recognition is how recall can be achieved when an object can have many different shapes, for example in handwriting. The simplest approach is to store many shapes for the same object, such as a single letter. But it is necessary to be able to recognize new instances of named objects, so, in addition to the individual representations that have already been described, generalized representations are also required. These are forms of storage that achieve recall in these circumstances. In the case where an object has no resemblance to previous examples a new shape would be stored, in effect it would be treated as a new shape with the same name but with a different stored representation.

The approach for generalized representations is to use multiple representations, which is storage of many shapes, but for parts of outlines that will be called basic objects. These are simple shapes that occur frequently, for example the parts of a single letter in handwriting that occur in one quadrant of the visual plane when the letter is at the visual centre. Use of multiple representations is one of the approaches identified by Tarr \& Pinker (1989) when investigating how orientation of an object influences recognition. Basic objects have shape and a $\boldsymbol{V H}$ position, i.e. they are not object-centred so the visual centre does not need to move to them. One way in which they could be obtained is by storage of slightly different shapes as viewpoint and distance change. The influence of stored basic objects may, perhaps, be experienced by observing a page of handwriting, first from the usual viewpoint, then with the page inverted.

\section{Representations of depth}

In three dimensions there is a requirement for a representation of depth, the distance coordinate perpendicular to the visual plane, increasing in the forward direction. For an object in the perception the size seen decreases as distance increases, but if there is awareness of depth then there can also be knowledge of its perceived actual size - that is an estimate of the size of the perpendicular projection $\mathbf{v}, \mathbf{h}$ onto the visual plane. In the experimental results of Foley, Ribeiro-Filho, \& Da Silva (2004) sizes were in the form of distances between stakes in a field; perceived size was described as "reported extent", and they pointed out that it may have been influenced by judgement, but that their results suggested that the effect was small. So perceived depth and actual size are probably less precise than $\tan (\psi), \tan (\varphi)$, the 
$\boldsymbol{V H}$ positions. In addition, the size seen is variable, apparently, because of the phenomenon of size "constancy" when depth changes during an observation. Another possibility, perhaps, is for size seen to be influenced by the size of the area of attention, increasing slightly as area decreases, and vice versa.

For a three dimensional object, depth in the model arises from rotation of a horizontal or vertical axis out of the visual plane, with a representation in the form of a projection $\mathbf{v} / \mathbf{D}, \mathbf{h} / \mathbf{D}$, onto the visual plane. The rotations of the axes are essential components in the procedure for recognition and knowledge of the object, even in the case of a new stored representation in current axes without recall, because axes would be used. Attentional planes are required for the rotations. The visual centre, which corresponds with the centre of the attentional plane, can be moved to various positions on the rotated object.

\section{Storage of shape representations of scenes}

Representations are within the overall area of attention, which includes all the objects in the scene. The representation contains $\boldsymbol{V H}$ positions of the centres of objects, in the coordinates of the overall area. As in the approach of Marr (1982), a modular organization is used, with object-centred coordinate systems here, for objects within the overall area, or for parts of an object stored separately as other objects. A difference in approach, however, is that in this model only rectangular coordinates are used, and representations are in two rather than three dimensions. Relative positions of objects are stored as coordinates of one origin of axes in the coordinates of the other, for example, the $\boldsymbol{V H}$ positions of the centre of an object in the coordinates of the overall area of attention as shown in Figure 16. The procedure starts by identification of an object, based on sizes of lengths of outline, largest first, followed by movement to the centre position of the object. In the case of scenes of connected objects centre positions are found by segmentation.

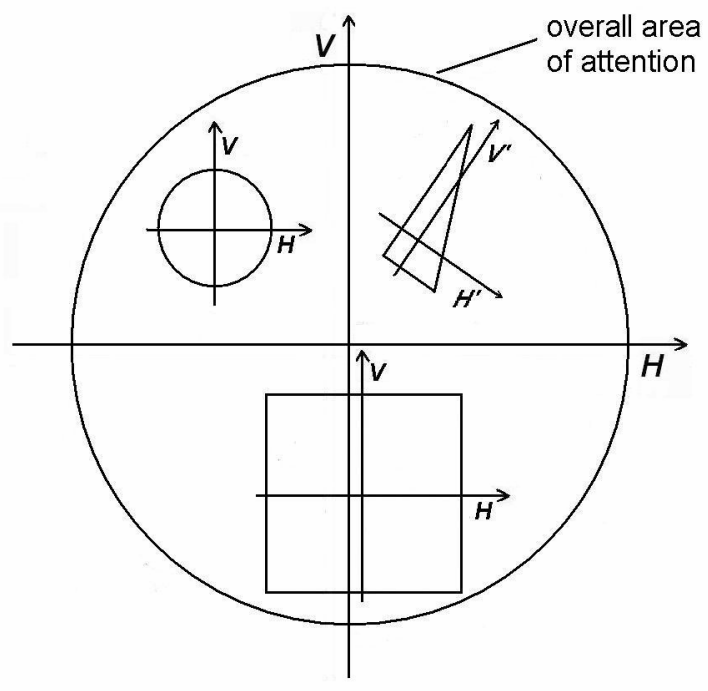

Figure 16 Storage of objects in a scene, using object-centred coordinates, within the overall area of attention. For recall, the visual centre would move to the centre of each object in turn.

Objects are stored at higher levels than the level of the overall area of the scene, with levels depending on the sizes of the objects; level of storage means the highest level stored at the centre position of an object 
There can be several different shifts in rotation within a single area of attention, if several objects had been stored in smaller areas - in effect, the early perception would be put into different axes.

\subsection{Segmentation}

Segmentation of an outline simplifies storage and recall, and involves setting the position and size of an area of attention. An object can be separated from a background by storing a representation only within the area of attention. Reduction in an area of attention progressively would exclude increasing amounts of background.

Position and size of the area are selected using ends of lengths, such that the distribution of their $\boldsymbol{V H}$ positions with respect to the centre of the area is balanced. The principle of "good continuation", see e.g. Bruce, Green \& Georgeson (2003), which is continuity of lengths of outline at junctions despite ends, seems to be a strong indication of shape for the purpose of segmentation.

\section{Discussion}

A number of additional points are included below.

\subsection{Detection of outlines and intensity}

Some experiments using stabilized retinal images (SRI) suggested that the perception fades over several seconds following onset of the stabilization. The method of outline detection used here is not consistent with those results, because it is necessary for fade to occur in a fraction of a second, probably in less than 100ms. In a review of work on eye movements, including SRI, Steinman \& Levinson (1990) concluded that there was some doubt whether stabilization had been achieved.

Because outlines are detected by change in intensity arising from movements, it is necessary to consider whether those could include tremor. At first sight tremor seems reasonable near the visual centre, because it is small in magnitude, however the frequency is high. Bolger et al. (1999) measured ocular microtremor, finding frequencies in the range $70-100 \mathrm{~Hz}$, with a mean frequency of $84 \mathrm{~Hz}$. If "on" or "off" responses can be produced by movements of outlines at this frequency, then tremor could be used for detection of sharp outlines, perhaps, with activity in the form of "spikes" corresponding with the "onset" or "offset" events.

\section{2 "On" and "off” responses}

In the model, a large response from a group indicates the presence of an outline, a smaller response represents intensity. Sharp outlines are detected in centres, blurred outlines in surrounds. The outlines would be "on" responses or "off" responses, depending on whether the movement of a group causing detection of outline were from a dark region to a lighter one, or from light to dark. For a particular direction of movement, sharp and blurred outlines, with the same sign of spatial change of intensity, would be detected in opposites of "on" and "off" cell types.

Responses representing outlines are transient, and are followed by sustained responses in the same cell, representing intensity. The representation will be in an "on" cell or an "off" cell, depending on whether the group moved across the outline into a relatively light or dark region. A consequence of this is that sustained activity in regions of uniform intensity would usually be of "on" or "off" type in the background, and the opposite type in the foreground. So, a wide range of intensity values in a scene would be divided between "on" and "off" responses, with the effect of splitting it into two smaller ranges. Whether or not a region appears "light" or "dark" would be determined by relative intensity. 


\subsection{Relative angles}

In Section 13 a description was given of angles of outline seen and relative angles stored. Within an object angles would be seen with reasonable precision relative to each other, but overall rotation of the object would be less precise. That would be the rotation of the attentional plane used for recall, with a precision based on primary increments. Usually attentional planes would be aligned with outlines. As pointed out by Schiffman (2001) p.186, an object can appear unchanged when the head is tilted $90^{\circ}$. By changing the attentional plane, a door frame can be still seen as vertical when the head is rotated $90^{\circ}$ sideways, or, by direction of attention elsewhere, with a rotation different from vertical. For scenes of many connected outlines it may sometimes be difficult to know the rotations of the attentional planes being used, and apparent angles of outline would depend on those rotations.

\subsection{Positions at edges of increments}

Useful insight may, perhaps, be gained from a deterministic model, in which case there is a question that is not satisfactorily resolved by use of overlapping groups. It is necessary to say how edges of primary increments influence recalled primary $\boldsymbol{V H}$ positions. In the perception seen the problem is avoided by use of traces, but a recalled detail in an outline could be close to the boundary between two increments. Another shape with the detail in a position only slightly different would not activate the required increment, but usual experience shows that it should be recalled as the same object. If a rule is applied that allows the new position to be different by a small amount, so that it is stored and included in an alternative representation of the object, then the same situation recurs when a subsequent observation finds the detail a further small distance away in the same direction. Repeated storage will cause the position of the detail to diverge from its original value.

Two features of the model may help to avoid this problem, namely, storage and recall in multiple levels, and a tendency to recall with less precision than the highest used for storage. Representations are stored in several levels, perhaps in many, with increments decreasing in size for each successively higher level. Boundaries between increments would in general be at different positions across the levels. Low levels are stored first, i.e. with an oversized area of attention. The size of the area is reduced progressively, storing more $\boldsymbol{V H}$ positions each time, perhaps up to a maximum between 10 and 20 radially. Larger numbers of positions require observations for larger periods of time. In the example described above, the detail would be included in a representation at a lower level producing recall of the object, with no need for storage of the new, slightly changed position.

\subsection{Spatial awareness and VH positions}

The value of a $\mathbf{V H}$ position is required to be proportional to the position $\mathbf{v} / \mathbf{D}, \mathbf{h} / \mathbf{D}$ in the visual plane, so that shape is maintained in the representation. There is a question concerning requirements for shape in the representation: whether or not it is sufficient for a stimulus of, say, three spots to cause activity at three cells in the network being modelled, and at the same cells every time the stimulus occurs. That would permit storage and recall of the "shape", but it would not be sufficient. A certain amount of knowledge would be stored, enabling recall, but there would be no knowledge of their spatial relationships - a representation is required for that kind of spatial awareness. Use of axes and coordinates allows that to be obtained.

So, spatial awareness arises from use of increment numbers as rectangular coordinates, with constant increments, approximately because of the precision of $\boldsymbol{V H}$ positions. The term "coordinates" here refers to the superposition of representations that can cause movements in the directions of $\boldsymbol{V}$ and $\boldsymbol{H}$. A single $\boldsymbol{V H}$ position is represented by a level and a pair of increment numbers, with each position having its own copy 
of the representation, to avoid shared increment numbers which do not define shapes adequately.

Should $\boldsymbol{V H}$ positions be called visual or motor activity representations?

- a $\boldsymbol{V H}$ position represents a position in the visual plane

- there is an ability to move the visual centre to a position in the visual plane

- they are obtained using movements - that links the positions in the visual plane with movements

- $\boldsymbol{V H}$ positions determine how motor activity representations are produced - they move to those positions

- one way to decide on the question, perhaps, is to say whether or not $\boldsymbol{V H}$ positions are used only for movements - if so, they would be motor activity representations; however, they are not used only in that way - they are the basis of the perception seen and stored shapes

- so, it can be argued that they are mainly visual representations, obtained using movements, and used to make movements to positions of interest

- the visual plane is imaginary, but describing $\boldsymbol{V H}$ positions as representations that define the direction, increment and extent of movements puts them into a more physical form.

\section{Conclusion}

A model for some of the basic procedures in perception and recognition of outlines has been described, using ideas and results most of which have been much discussed in the literature. Many topics in the study of vision are more than "basic", but the aim was to provide a model that does not obviously preclude them. As progress is made towards a satisfactory understanding of vision, it is likely that most of the subjects addressed here will have to be considered, if only so that they can be included or excluded. If they are included, one way in which they can be combined has been given in the description above.

\section{References}

Ahissar, E., \& Arieli, A. (2001). Figuring space by time. Neuron. 32, 185-201.

Ahissar, E., \& Arieli, A. (2012). Seeing via miniature eye movements: a dynamic hypothesis for vision. Frontiers in Computational Neuroscience. 6, Article 89, 1-27.

Biederman, I. (1987). Recognition-by-components: A theory of human image understanding. Psychological Review. 94, 115-147.

Bolger, C., Bojanic, S., Sheahan, N.F., Coakley, D., \& Malone, J.F. (1999). Dominant frequency content of ocular microtremor from normal subjects. Vision Research. 39, 1911-1915.

Bruce, V., Green, P.R., \& Georgeson, M.A. (2003). Visual Perception, Physiology, psychology, \& ecology, fourth ed. Psychology Press, Hove and New York.

Foley, J.M., Ribeiro-Filho, N.P., \& Da Silva, J.A. (2004). Visual perception of extent and the geometry of visual space. Vision Research. 44, 147-156.

Gross, R. (2001). Psychology - The Science of Mind and Behaviour. Hodder \& Stoughton.

Helmholtz, H. von. (1867). Translated from the third German edition, Southall, J.P.C. (Ed.) (1925), Helmholtz's Treatise on Physiological Optics Vol. 3, Electronic edition (2001): University of Pennsylvania, URL:http//psych.upenn.edu/backuslab/helmholtz

Howard, I.P. (1982). Human Visual Orientation. John Wiley \& Sons, Chichester and New York. 
Hummel, J.E., \& Biederman, I. (1992). Dynamic binding in a neural network for shape recognition. Psychological Review. 99, 480-517.

Marr, D. (1982). Vision: A computational investigation into the human representation and processing of visual information. W.H. Freeman \& Co., New York.

Pinker, S. (1984). Visual cognition: An introduction. Cognition. 18, 1-63.

Schiffman, H.R. (2001). Sensation and Perception, An integrated approach, fifth ed. John Wiley \& Sons, Chichester and New York.

Steinman, R.M., \& Levinson, J.Z. (1990). The role of eye movement in the detection of contrast and spatial detail, in: Kowler, E., (Ed.), Eye Movements and their Role in Visual and Cognitive Processes. Elsevier Science Publishers BV, chapter 3, pp. 115-212.

Tarr, M.J., \& Pinker, S. (1989). Mental rotation and orientation-dependence in shape recognition. Cognitive Psychology. 21, 233-282. 